\title{
Fermion masses and quantum numbers from extra dimensions.
}

\author{
Andrey Neronov \\ Theoretische Physik, Universität München, Theresienstr., 37, 80333, Munich, Germany
}

\begin{abstract}
We study the localization of fermions on a brane embedded in a space-time with $A d S_{n} \times M^{k}$ geometry. Quantum numbers of localized fermions are associated with their rotation momenta around the brane. Fermions with different quantum numbers have different higher-dimensional profiles. Fermion masses and mixings, which are proportional to the overlap of higher-dimensional profiles of the fermions, depend on the fermion quantum numbers.
\end{abstract}

\section{INTRODUCTION.}

The problem of explaining the hierarchy of fermion masses and mixings is one of the longstanding problems of particle physics which can not be resolved within the standard model [ []. This problem becomes especially interesting in the light of recent results on nonzero neutrino masses [2]. There exist different approaches to explanation of the hierarchy of fermion masses, like models with additional "horizontal" symmetry [3], or models where the hierarchy is generated by the "seesaw" mechanism [4], the last class of models is particularly popular for explanation of smallness of neutrino masses. Apart from the seesaw mechanism the smallness of neutrino masses can be related to the possible existence of large extra dimensions [5]. The idea is to assume that the right-handed sterile neutrino can propagate in higher-dimensional bulk while the left-handed neutrinos are confined to a four-dimensional brane so that the higher-dimensional profiles of left and right neutrinos have small overlap. Actually, it is possible to relate the whole hierarchy of fermion masses and mixings to the differences in the overlaps of higher-dimensional fermion profiles [6.7].

What can be the reason for different fermions to have different higher-dimensional profiles? What mechanism can be responsible for the fact that a fermion which is neutral with respect to the $S U(3) \times S U(2) \times U(1)$ group of the standard model is not localized on the brane?

If a brane is embedded as a surface in a higher-dimensional space, matter fields bound to the brane are naturally classified by the values of their momenta of rotation around the brane. Indeed, in quite general settings, the space-time around the brane possess a rotation symmetry. For example, in the case of just two extra dimensions the metric around the brane has the form

$$
d s^{2}=e^{\nu(\rho)} \eta_{\mu \nu} d x^{\mu} d x^{\nu}+e^{\lambda(\rho)} d \rho^{2}+e^{\mu(\rho)} d y^{2}
$$

where $\eta_{\mu \nu}=\operatorname{diag}(-1,1,1,1)$ is the four-dimensional Minkowsky metric and functions $\nu(\rho), \lambda(\rho), \mu(\rho)$ are determined from Einstein equations. The coordinate $\rho$ measures the 
distance from the brane placed at $\rho=0$ and the coordinate $y$ is periodic $y \in\left[0,2 \pi R_{y}\right)$. The metric (1.1) possesses a $U(1)_{y}$ symmetry of rotations in $y$ direction

$$
y \rightarrow y+\text { const }
$$

As in usual quantum mechanics, matter fields $\Psi$ bound to the brane are characterized by different values $q$ of the rotation momentum in the $y$ direction

$$
\Psi_{q}(\rho, y)=F(\rho) e^{i q y}
$$

From the four-dimensional point of view, this rotation momentum is a quantum number of observable four-dimensional particles. The profiles $\Psi_{q}(\rho, y)$ ("wave functions") of the bound states depend on the values of the rotation momentum. This means that particles with different quantum numbers $q$ have different higher-dimensional profiles [8].

In what follows we consider branes embedded into higher-dimensional spaces with (asymptotic) $A d S_{n} \times M^{k}$ geometry of direct product of $n$-dimensional anti-deSitter space and compact manifold $M^{k}$. Such spaces arise naturally in Freund-Rubin compactifications [9] of higher-dimensional supergravities [10] and received a considerable attention recently after the discovery of $A d S / C F T$ correspondence [11] and observation that gravity can be localized on a brane in five-dimensional [12] or higher-dimensional [13] anti-deSitter space.

In section [1] we show that in six-dimensional space (1.1) fermion zero modes which are charged with respect to $U(1)_{y}$ group of rotations in $y$ direction (1.2) can be localized on the brane, while the state neutral with respect to $U(1)_{y}$ propagates in the bulk. In Section III we discuss the breaking of rotation symmetry $U(1)_{y}(1.2)$ and derive a formula relating the effective four-dimensional mass of a localized fermion state and charges $q_{L}$ and $q_{R}$ of its left and right handed components. We also consider the case when the brane is embedded in seven-dimensional space with $A d S_{7}$ geometry. In this case localized fermions carry $U(1) \times U(1)$ charges. The extra $U(1)$ symmetry can be associated with the "horizontal" symmetry [3] needed to distinguish between fermions from different generations. We show that in this model the masses of localized fermions are arranged hierarchically. In Section IV we consider a more complicated model where the localized fermions carry $S U(2) \times U(1)$ quantum numbers. In this case the brane is embedded into space-time which becomes the direct product of $A d S$ space with a two-dimensional sphere $S^{2}$ far away from the brane. We find the higher-dimensional profiles of $S U(2)$ singlet and doublet states localized on the brane. If the rotation symmetry $S U(2)$ is broken, effective four-dimensional mixing between singlet and doublet states becomes nonzero and one of the doublet components becomes massive. The other component ("left-handed neutrino") is not mixed with the states localized on the brane and, therefore, its mass is naturally small.

\section{FERMION QUANTUM NUMBERS AS MOMENTA OF ROTATION AROUND THE BRANE.}

Let us consider a brane embedded in the space-time (1.1). Suppose that all the matter fields are localized in a region $0 \leq \rho \leq \rho_{0}$ while the metric outside the brane, $\rho_{0}<\rho<\infty$, is a solution of vacuum Einstein equations, possibly with a cosmological constant term. 
In the most simple case the bulk metric is isometric to the metric of the six-dimensional anti-deSitter space $A d S_{6}$

$$
d s^{2}=\frac{1}{(\kappa \rho)^{2}}\left(\eta_{\mu \nu} d x^{\mu} d x^{\nu}+d \rho^{2}+d y^{2}\right)
$$

where $\kappa$ is the inverse curvature radius of anti-deSitter space.

The massless modes of higher-dimensional Dirac field can be naturally localized on the brane in a space-time (1.1) with nontrivial warp factors $\nu(\rho), \lambda(\rho), \mu(\rho)[\mathbb{8}$. In order to see this let us consider the higher-dimensional Dirac equation

$$
\Gamma^{A} D_{A} \Psi=0
$$

The six-dimensional gamma matrices $\Gamma^{A}$ are defined with the help of the vielbein $E_{\hat{B}}^{A}$ and flat space gamma matrices $\Gamma^{\hat{A}}$

$$
\Gamma^{A}=E_{\hat{B}}^{A} \Gamma^{\hat{B}}
$$

(the indexes with a hat are six-dimensional Lorentz indexes). The covariant derivative is defined as

$$
D_{A} \Psi=\Psi_{, A}+\frac{1}{2} \omega_{A}^{\hat{B} \hat{C}} \sigma_{\hat{B} \hat{C}} \Psi
$$

where $\omega_{A}^{\hat{B} \hat{C}}$ is the spin connection expressed through the vielbein $E_{\hat{B}}^{A}$ and $\sigma_{\hat{B} \hat{C}}=\frac{1}{4}\left[\Gamma_{\hat{B}} \Gamma_{\hat{C}}\right]$ are generators of the six-dimensional Lorentz group. Taking the coordinate vielbein for the metric $(1.1)$

$$
\begin{aligned}
& E_{\mu}^{\hat{\alpha}}=e^{\nu / 2} \delta_{\mu}^{\hat{\alpha}} \\
& E_{\rho}^{\hat{\rho}}=e^{\lambda / 2} \\
& E_{y}^{\hat{y}}=e^{\mu / 2}
\end{aligned}
$$

we find

$$
\frac{1}{2} \omega_{A}^{\hat{B} \hat{C}} \Gamma^{A} \sigma_{\hat{B} \hat{C}}=e^{-\lambda / 2}\left(\nu^{\prime}+\frac{\mu^{\prime}}{4}\right) \Gamma^{\hat{r}}
$$

where the prime denotes the $\rho$ derivative. Substituting the covariant derivative (2.4) into the Dirac equation (2.2) we find

$$
e^{-\nu / 2} \Gamma^{\hat{\mu}} \Psi_{, \mu}+e^{-\lambda / 2} \Gamma^{\hat{r}}\left(\Psi^{\prime}+\left(\nu^{\prime}+\frac{\mu^{\prime}}{4}\right) \Psi\right)+e^{-\mu / 2} \Gamma^{\hat{y}} \Psi_{, y}=0
$$

The six-dimensional spinor $\Psi$ can be decomposed on a four-component spinor $\psi$ and twocomponent spinor $\tilde{\Psi}$ :

$$
\Psi=\psi(x) \otimes \tilde{\Psi}(r, y)
$$

If we are interested in the massless solutions of Dirac equation, the four-dimensional spinor $\psi$ satisfies 


$$
\Gamma^{\hat{\mu}} \psi_{, \mu}=0
$$

and the equation (2.8) reduces to

$$
\Gamma^{\hat{r}}\left(\tilde{\Psi}^{\prime}+\left(\nu^{\prime}+\frac{\mu^{\prime}}{4}\right) \tilde{\Psi}\right)+e^{(\lambda-\mu) / 2} \Gamma^{\hat{y}} \tilde{\Psi}_{, y}=0
$$

We can expand the solutions over the states with fixed rotation momentum $q_{y}$ in the $y$ direction

$$
\tilde{\Psi}=e^{-\nu-\mu / 4} \exp \left\{\frac{i q_{y} y}{R_{y}}\right\} F(\rho)
$$

( $F$ is a two-component spinor). If we take the two gamma matrices $\Gamma^{\hat{r}}$ and $\Gamma^{\hat{y}}$ to be

$$
\Gamma^{\hat{r}}=\left(\begin{array}{cc}
0 & 1 \\
1 & 0
\end{array}\right) ; \quad \Gamma^{\hat{y}}=\left(\begin{array}{cc}
0 & -i \\
i & 0
\end{array}\right)
$$

we find that the two-component spinor $F=(f, g)$ satisfies the equations

$$
\begin{aligned}
f^{\prime}-e^{(\lambda-\mu) / 2} \frac{q_{y}}{R_{y}} f & =0 \\
g^{\prime}+e^{(\lambda-\mu) / 2} \frac{q_{y}}{R_{y}} g & =0
\end{aligned}
$$

Thus, if $q_{y}>0$ and the bulk metric is isometric to anti-deSitter metric (2.1), the spinor

$$
\Psi_{q}=\sqrt{\frac{q_{y}}{\pi}} \frac{(\kappa \rho)^{5 / 2}}{R_{y}}\left(\exp \left\{\frac{i q_{y} y}{R_{y}}-\frac{q\left(\rho-\rho_{0}\right)}{R_{y}}\right\}\right) \otimes \psi(x)
$$

describes a fermion state bound to the brane (We postpone the discussion of the behavior of solutions of Dirac equation in the core of the brane $0 \leq \rho \leq \rho_{0}$ till Section (IV). We have normalized the solution (2.15) with respect to the scalar product

$$
\left\langle\Psi_{q}, \Psi_{q}\right\rangle=\int d^{4} x d \rho d y \sqrt{-g} \bar{\Psi}_{q} \Gamma^{0} \Psi_{q}
$$

The rotation momentum $q_{y}$ is, in fact, a charge of the localized fermion mode with respect to Kaluza-Klein gauge field $A_{\mu}$ which corresponds to the $U(1)_{y}$ symmetry (11.2) of the metric (1.1). Indeed, the metric (1.1) possesses a Killing vector

$$
K=\partial_{y}
$$

which, according to Kaluza-Klein mechanism leads to the existence of a gauge field $A_{\mu}$ in effective four-dimensional theory. This gauge field arises as a nondiagonal component

$$
d s^{2}=e^{\nu} \eta_{\mu \nu} d x^{\mu} d x^{\nu}+e^{\lambda} d \rho^{2}+e^{\mu}\left(d y+A_{\mu} d x^{\mu}\right)^{2}
$$

of higher-dimensional gravitational perturbations. The zero mode of the field $A_{\mu}$ can be localized on the brane [8]. In this case $A_{\mu}$ can correspond to an observable $U(1)$ gauge field and the charge $q_{y}$ can be related to an observable quantum number of standard model fermions. Note that the $U(1)_{y}$-neutral state with $q_{y}=0$ ("sterile neutrino") is not localized on the brane. 


\section{BREAKING OF $U(1)$ ROTATION SYMMETRY AND GENERATION OF FERMION MASSES.}

We are interested in mixings between differently charged fermions $\Psi_{q_{1}}$ and $\Psi_{q_{2}}$

$$
S_{m i x} \sim f \int d^{4} x d \rho d y \sqrt{-g} \bar{\Psi}_{q_{1}} \mathcal{O}_{q_{1} q_{2}} \Psi_{q_{2}}
$$

here $\mathcal{O}_{q_{1} q_{2}}$ is a matrix with indexes which run through all fermion species (in our example through all possible $q_{y}$ ) and $f$ is a constant. Let us consider (3.1) in more details. Higherdimensional profiles (2.15) of the localized fermion zero modes $\Psi_{q}$ depend on their charges $q_{y}$. The mixing (3.1) between modes with charges $q_{1}$ and $q_{2}$ is proportional to the integral over the extra dimensions of the overlap of the profiles of $\Psi_{q_{1}}$ and $\Psi_{q_{2}}$. This integral, in turn includes the integral over the circle $S^{1}$ parameterized by the coordinate $y$. Substituting the profiles (2.12) into (3.1) we find

$$
S_{m i x} \sim f \int d \rho \int_{0}^{2 \pi R_{y}} d y \sqrt{-g} e^{i\left(q_{2}-q_{1}\right) y / R_{y}} \bar{F}_{q_{1}}(r) F_{q_{2}}(r)
$$

It is not difficult to see that this integral vanishes if $q_{1} \neq q_{2}$ since the modes with different $q_{y}$ are orthogonal to each other. Thus, when the symmetry of rotations around the brane is not broken, the mixings between the modes with different charges vanish.

Suppose, that the symmetry of rotations $y \rightarrow y+$ const is broken by some mechanism. We do not discuss here different possibilities for particular mechanism of symmetry breaking in the context of theories with extra dimensions (see, for example [14]). The symmetry breaking results in appearance of a (fundamental or effective) Higgs field which has nonzero $U(1)_{y}$ charge $p$

$$
H_{p}=H_{p}(\rho) \exp \left\{\frac{i p y}{R_{y}}\right\}
$$

The Higgs field is coupled to the higher-dimensional Dirac field

$$
S_{m i x} \sim f \int d^{4} x d \rho d y \sqrt{-g} H_{p} \bar{\Psi}_{q_{1}} \Psi_{q_{2}}
$$

Substituting the profiles (2.15), (3.3) into (3.4) we find that the mixing between the modes $\Psi_{q_{1}}, \Psi_{q_{2}}$ with the charges $q_{1}, q_{2}$ such that

$$
p-q_{1}+q_{2}=0
$$

does not vanish.

Using the decomposition of the fermion field (2.9) we can write the mixing (3.4) in the form

$$
S_{\text {mix }} \sim M_{q_{1} q_{2}} \int d^{4} x \bar{\psi}_{q_{1}}(x) \psi_{q_{2}}(x)
$$

with 


$$
M_{q_{1} q_{2}}=f \int d \rho d y \sqrt{-g} H_{p} \tilde{\bar{\Psi}}_{q_{1}} \tilde{\Psi}_{q_{2}}
$$

The mixing $M_{q_{1} q_{2}}$ can be naturally small, if the higher-dimensional profiles of fermions $\Psi_{q_{1}}, \Psi_{q_{2}}$ have small overlap with each other, or with the Higgs profile $H_{p}$.

The Dirac mass of a fermion is the mixing between its left and right handed components $\Psi_{L}$ and $\Psi_{R}$. If the charges of $\Psi_{L}, \Psi_{R}$ are respectively $q_{L}$, and $q_{R}=q_{L}+p$, we get from (3.7)

$$
M_{D}=f \int d \rho d y e^{2 \nu+\lambda / 2+\mu / 2} H_{p} \tilde{\bar{\Psi}}_{q_{L}} \tilde{\Psi}_{q_{R}}
$$

Since we do not discuss the details of the symmetry breaking, we can not calculate the Higgs profile $H_{p}(\rho)$. Let us consider two extreme possibilities. If the profile $H_{p}(\rho)$ is "smooth",

$$
H_{p}(\rho) \approx h=\text { const }
$$

we get, substituting the profiles (2.15) into (3.8) and performing the integral

$$
M_{D}=\frac{2 f h \sqrt{q_{L} q_{R}}}{\kappa R_{y}} e^{\left(q_{L}+q_{R}\right) \rho_{0} / R_{y}} \Gamma\left(0, \frac{\left(q_{L}+q_{R}\right) \rho_{0}}{R_{y}}\right)
$$

where $\Gamma(0, x)$ is the incomplete Gamma-function. (We have supposed that the space-time metric outside the brane is the anti-deSitter metric (2.1). $\rho_{0}$ is the thickness of the brane core.) If the brane thickness $\rho_{0}$ is much larger than $R_{y}$ we get an approximate expression

$$
M_{D} \approx \frac{2 f h}{\kappa \rho_{0}} \frac{\sqrt{q_{L} q_{R}}}{\left(q_{L}+q_{R}\right)}
$$

If the profile $H_{p}(\rho)$ of the Higgs field is "sharp", that is peaked at a distance $\rho_{h}$ from the center of the brane

$$
H_{p}(\rho) \approx h \rho_{h} \delta\left(\rho-\rho_{h}\right)
$$

we get from $(3.8)$

$$
M_{D}=\frac{2 f h \sqrt{q_{L} q_{R}}}{\kappa R_{y}} e^{-\left(q_{L}+q_{R}\right)\left(\rho_{h}-\rho_{0}\right) / R_{y}}
$$

In this case, if $\rho_{h}-\rho_{0}=($ several $) R_{y}$, the masses of particles with different $q_{L}, q_{R}$ are arranged hierarchically. The fact that the sharp higher-dimensional profile of the Higgs field leads to a hierarchical structure of four-dimensional fermion masses was noted in [0, [15].

As a simple generalization of the above model let us consider a brane embedded in a seven-dimensional space with $A d S_{7}$ geometry

$$
d s^{2}=\frac{1}{(\kappa \rho)^{2}}\left(\eta_{\mu \nu} d x^{\mu} d x^{\nu}+d y^{2}+d z^{2}+d \rho^{2}\right)
$$

The seven-dimensional anti-deSitter space can be obtained after compactification of elevendimensional supergravity [9]. Suppose that, as in the above example (1.1) the coordinate $\rho$ counts the distance from the brane and the coordinates $y$ and $z$ are periodic with the periods 
$2 \pi R_{y}, 2 \pi R_{z}$. The symmetry group of rotations around the brane is now $U(1)_{y} \times U(1)_{z}$ - the product of rotations in $y$ and $z$ directions. The fermions bound to the brane possess two quantum numbers $q_{y}, q_{z}$ which are rotation momenta in $y$ and $z$ directions

$$
\Psi=\exp \left\{\frac{i q_{y} y}{R_{y}}+\frac{i q_{z} z}{R_{z}}\right\} F(r) \otimes \psi(x)
$$

The Dirac equation for zero modes $\Gamma^{\hat{\mu}} \psi_{, \mu}=0$ is reduced to

$$
\Gamma^{\hat{r}}\left(F^{\prime}-\frac{5}{\rho} F\right)+\frac{i q_{y}}{R_{y}} \Gamma^{\hat{y}} F+\frac{i q_{z}}{R_{z}} \Gamma^{\hat{z}} F=0
$$

It has normalized solutions

$$
F_{q_{y}, q_{z}}=\frac{\kappa^{3} \rho^{3} e^{-Q\left(\rho-\rho_{0}\right)}}{2 \sqrt{2} \pi R_{y} R_{z}\left(q_{z}^{2} R_{y}^{2}+q_{y}^{2} R_{z}^{2}\right)^{1 / 4}}\left(\begin{array}{r}
\sqrt{q_{z}^{2} R_{y}^{2}+q_{y}^{2} R_{z}^{2}} \\
q_{z} R_{y}+i q_{y} R_{z}
\end{array}\right)
$$

where

$$
Q=\sqrt{\frac{q_{y}^{2}}{R_{y}^{2}}+\frac{q_{z}^{2}}{R_{z}^{2}}}
$$

The Dirac mass $M_{D}$ which is the mixing between the modes $\Psi_{q_{y L}, q_{z L}}$ and $\Psi_{q_{y R}, q_{z R}}$ is given by the integral over the extra dimensions of the overlap of the profiles of left and right handed components with the profile of the Higgs field (see (3.7)). In the case of the sharp profile (3.12) of the Higgs field $M_{D}$ is proportional to

$$
M_{D} \sim \exp \left\{-\left(\sqrt{\frac{q_{y L}^{2}}{R_{y}^{2}}+\frac{q_{z L}^{2}}{R_{z}^{2}}}-\sqrt{\frac{q_{y R}^{2}}{R_{y}^{2}}+\frac{q_{z R}^{2}}{R_{z}^{2}}}\right)\left(\rho_{h}-\rho_{0}\right)\right\}
$$

(compare with (3.13)). If the scales $R_{y}, R_{z}$ and $\left(\rho_{h}-\rho_{0}\right)$ are arranged as $R_{y} \sim($ several $) R_{z}$, $\left(\rho_{h}-\rho_{0}\right) \sim($ several $) R_{z}$, the masses of particles with different $q_{z L}, q_{z R}$ go as different powers of a small parameter

$$
M_{D} \sim \epsilon^{\left(q_{z L}-q_{z R}\right)}
$$

where $\epsilon$ is

$$
\epsilon=\exp \left\{-\frac{\rho_{h}-\rho_{0}}{R_{z}}\right\}
$$

The rotation symmetry $U(1)_{z}$ can be identified with the "horizontal" symmetry [3] which is introduced in some approaches to the fermion mass hierarchy and enables to distinguish between fermions from different generations. The mass hierarchy of Eq. (3.20) is similar to the formula for the fermion masses derived in the models with the horizontal symmetry. In order to get a realistic pattern of masses of the standard model fermions the parameter $\epsilon$ must have numerical value

$$
\epsilon \approx 0.049
$$


which means that, if the thickness of the brane $\rho_{0}$ is negligibly small, the relation between the radius of the Higgs orbit $r_{h}$ and the size of the circle $S^{1}$ parameterized by the coordinate $z$ must be

$$
\rho_{h} \approx 3 R_{z}
$$

From (3.19) one can see that the masses of fermions with different charges $q_{y L}, q_{y R}$ are quasidegenerate.

\section{A MODEL WITH $S U(2) \times U(1)$ SYMMETRY.}

Up to now we have considered models in which fermions carry only $U(1)$ charges. If we want to include $S U(2) \sim S O(3)$ as a symmetry group of rotations around the brane, a natural generalization of the model of the previous sections would be to consider a brane embedded in an eight-dimensional space

$$
d s^{2}=e^{\nu(r)} \eta_{\mu \nu} d x^{\mu} d x^{\nu}+e^{\mu(r)} d y^{2}+e^{\lambda(r)}\left(d r^{2}+r^{2}\left(d \theta^{2}+\sin ^{2} \theta d \phi^{2}\right)\right)
$$

that is, to add two more extra dimensions $(\theta, \phi)$ with the geometry of two-dimensional sphere.

In the previous sections we have systematically abandoned the discussion of behavior of the fermion zero modes in the core of the brane. At the same time, if the brane is considered as a topological defect in a higher-dimensional space-time, the analysis of behavior of fermion zero modes in the core of defect is important, because the requirement of regularity of the fermion profiles in the core can impose restrictions on the number of normalizable zero modes bound to the brane [16]. In this section we will extend the analysis of fermion zero modes to the brane core. The topology of the core of the brane embedded into eight-dimensional space-time (4.1) depends on the behavior of warp factors $\nu(r), \lambda(r), \mu(r)$ in the limit $r \rightarrow 0$ [17]. We consider the situation when

$$
\nu(r), \mu(r), \lambda(r) \rightarrow 0, \quad r \rightarrow 0
$$

so that the topology of the brane core is $R^{4} \times S^{1} \times D^{3}$, where $D^{3}$ is the three-dimensional disk $(r, \theta, \phi), 0 \leq r \leq r_{0}$.

The solutions of the eight-dimensional Dirac equation can be expanded over the eigenstates of the total angular momentum

$$
\vec{L}=\vec{r} \times \vec{p}+\frac{1}{2} \vec{\sigma}
$$

Here $\vec{r} \times \vec{p}$ is the usual angular momentum in three-dimensional space $(r, \theta, \phi)$ and $\frac{1}{2} \vec{\sigma}$ is the spin operator, $\left(\sigma^{i}\right.$ are the Pauli matrices). If we relate $S U(2)$ symmetry of space-time (4.1) to the $S U(2)_{L}$ gauge group of the standard model, we face immediately the following difficulty. It is known, that the right-handed fermions of the standard model are singlet representations of $S U(2)_{L}$. But the lowest possible value of the angular momentum (4.3) is

$$
l_{\min }=1 / 2 .
$$


The state with the lowest angular momentum is a doublet representation of $S U(2)$.

This difficulty can be resolved if we suppose that the space-time (4.1) is obtained in result of Freund-Rubin compactification [9] of higher-dimensional theory. For example, the space-time with geometry

$$
d s^{2}=\frac{1}{\kappa^{2} r^{2 \kappa \mathcal{R}}}\left(\eta_{\mu \nu} d x^{\mu} d x^{\nu}+d y^{2}\right)+\frac{\mathcal{R}^{2}}{r^{2}}\left(d r^{2}+r^{2}\left(d \theta^{2}+\sin ^{2} \theta d \phi^{2}\right)\right)
$$

of direct product $A d S_{6} \times S^{2}$ of anti-deSitter space with a two-sphere of the radius $\mathcal{R}$ can be obtained as a solution of eight-dimensional Einstein-Maxwell equations if we introduce a vector field $\mathcal{A}_{M}$ in a topologically nontrivial monopole-like configuration

$$
\mathcal{A}_{\phi}=m(1-\cos \theta)
$$

in the eight-dimensional bulk. The metric (4.5) can describe the space-time geometry outside the brane, $r_{0}<r$, or asymptotically when $r \rightarrow \infty$, while in the core of the brane, $0 \leq r \leq r_{0}$, the metric is a solution of the Einstein-Maxwell equations coupled to other matter fields and the asymptotic behavior of the metric in the $r \rightarrow 0$ limit is given by (4.2).

If the higher-dimensional spin-1/2 field $\Psi$ has nonzero charge $e$ with respect to $\mathcal{A}_{M}$, the conserved total angular momentum is

$$
\vec{J}=\vec{r} \times \vec{p}+\frac{1}{2} \vec{\sigma}-e m \frac{\vec{r}}{r}
$$

rather then (4.3). The lowest possible value of the angular momentum is

$$
j_{\text {min }}=|e m|-1 / 2
$$

and if $|e m|=1 / 2$ the state with the lowest angular momentum is the $S U(2)$ singlet. The properties of the fermion zero modes in the model with monopole-like configuration (4.6) of the vector field $\mathcal{A}_{M}$ on the two-sphere $S^{2}$ were first considered in 18 .

In fact, the existence of a fundamental vector field $\mathcal{A}_{M}$ in the eight-dimensional bulk is not a necessary condition for the existence of $S U(2)$ singlets in the spectrum of fermionic modes. If we consider a little bit more complicated Ansatz for the background metric in the eight-dimensional space-time

$$
d s^{2}=e^{\nu(r)} \eta_{\mu \nu} d x^{\mu} d x^{\nu}+e^{\mu(r)}\left(d y+\mathcal{A}_{\phi} d \phi\right)^{2}+e^{\lambda(r)}\left(d r^{2}+r^{2}\left(d \theta^{2}+\sin ^{2} \theta d \phi^{2}\right)\right)
$$

where $\mathcal{A}_{\phi}$ is given by (4.6), the conserved angular momentum is again given by (4.7). The only difference between the above two ways of introducing monopole-like configuration (4.6) in the higher-dimensional theory is that in the Kaluza-Klein case (4.9) the Dirac field has a nominimal coupling to the field $\mathcal{A}_{M}$ [19]:

$$
L_{i n t} \sim \frac{m}{4 r^{2}} e^{\mu / 2-\lambda} \bar{\Psi} \Gamma^{\hat{\theta}} \Gamma^{\hat{\phi}} \Gamma^{\hat{y}} \Psi
$$

and the charge $e$ is expressed through the rotation momentum in $y$ direction

$$
e=\frac{q_{y}}{R_{y}}
$$


In both cases (in the space-time (4.1) with the fundamental vector field $\mathcal{A}_{M}$ or in the spacetime (4.9) with Kaluza-Klein vector field $\mathcal{A}_{M}$ ) the Dirac equation for the fermion zero modes $\left(\Gamma^{\hat{\mu}} \Psi_{, \mu}=0\right)$ reduces to

$$
\begin{gathered}
e^{-\lambda / 2}\left[\Gamma^{\hat{r}}\left(\Psi^{\prime}+\left(\nu^{\prime}+\frac{\mu^{\prime}}{4}+\frac{\lambda^{\prime}}{2}+\frac{1}{r}\right) \Psi\right)+\frac{1}{r} \Gamma^{\hat{\theta}}\left(\Psi, \theta+\frac{1}{2} \cot \theta \Psi\right)\right. \\
\left.+\frac{1}{r \sin \theta} \Gamma^{\hat{\phi}}\left(\Psi_{, \phi}-i e \mathcal{A}_{\phi} \Psi\right)\right]+e^{-\mu / 2} \Gamma^{\hat{y}} \Psi_{, y}+k \frac{m}{4 r^{2}} e^{\mu / 2-\lambda} \Gamma^{\hat{\theta}} \Gamma^{\hat{\phi}} \Gamma^{\hat{y}} \Psi=0
\end{gathered}
$$

(we have introduced an arbitrary coefficient $k$ in front of the nonminimal coupling term in order to be able to analyze the cases with and without nonminimal coupling (4.10) simultaneously). It is convenient to write $\Psi$ in the form

$$
\Psi_{q}=\frac{1}{r} \exp \left\{-\nu-\frac{\mu}{4}-\frac{\lambda}{2}\right\} \exp \left\{\frac{i q_{y} y}{R_{y}}\right\} e^{-i \Gamma^{\hat{y}}} F(r, \theta, \phi)
$$

where $F$ is a four-component spinor. From (4.12) we find that $F$ is a zero-energy solution of the four-dimensional Dirac equation

$$
H F=0
$$

with the Hamiltonian

$$
H=\vec{\alpha}(\vec{p}-e \vec{A})+\beta \frac{q_{y}}{R_{y}} e^{(\lambda-\mu) / 2}-k \frac{m}{4 r^{2}} e^{(\mu-\lambda) / 2} \beta \frac{(\vec{\sigma} \vec{r})}{r^{3}}
$$

Here the matrices $\vec{\alpha}$ and $\beta$ are

$$
\vec{\alpha}=\left(\begin{array}{cc}
0 & \vec{\sigma} \\
\vec{\sigma} & 0
\end{array}\right), \beta=\left(\begin{array}{cc}
1 & 0 \\
0 & -1
\end{array}\right)
$$

The Hamiltonian (4.15) coincides with the Hamiltonian of Dirac particle in the central field

$$
V(r)=\frac{q_{y}}{R_{y}} e^{(\lambda-\mu) / 2}
$$

superposed with the field of magnetic monopole (4.6). The last term in the Hamiltonian (4.15) describes an extra magnetic moment of Dirac particle. The bound states of Dirac particles with an extra magnetic moment in the field of magnetic monopole were studied by Kazama and Yang [20]. The state with the lowest value (4.8) of the total angular momentum (4.7) and its projection $J_{3}=m$ on some axis is described by the spinor

$$
F=\left(\begin{array}{c}
f(r) \\
i g(r)
\end{array}\right) \otimes \eta_{|e m|, j, m}(\theta, \phi)
$$

where $\eta_{|e m|, j, m}$ is the two-component spinor

$$
\eta_{(e g), j, m}=\left(\begin{array}{c}
-\sqrt{\frac{j-m+1}{2 j+2}} Y_{(e g), j+1 / 2, m-1 / 2} \\
\sqrt{\frac{j+m+1}{2 j+2}} Y_{(e g), j+1 / 2, m+1 / 2}
\end{array}\right)
$$


and $Y_{q, j, m}(\theta, \phi)$ are the monopole harmonics [21]. Substituting (4.18) into (4.14) we find that functions $f(r), g(r)$ satisfy equations

$$
\begin{aligned}
& f^{\prime}=\left(\frac{q_{y}}{R_{y}} e^{(\lambda-\mu) / 2}-k \frac{m}{4 r^{2}} e^{(\mu-\lambda) / 2}\right) g \\
& g^{\prime}=\left(\frac{q_{y}}{R_{y}} e^{(\lambda-\mu) / 2}-k \frac{m}{4 r^{2}} e^{(\mu-\lambda) / 2}\right) f
\end{aligned}
$$

We are interested in the bound state solution

$$
f=-g=C \exp \left\{-\int d r\left(\frac{q_{y}}{R_{y}} e^{(\lambda-\mu) / 2}-k \frac{m}{4 r^{2}} e^{(\mu-\lambda) / 2}\right)\right\}
$$

( $C$ is a normalization constant). Suppose that at large $r$ the bulk metric approaches the metric (4.5) of $A d S_{6} \times S^{2}$ space. Then the asymptotic behavior of (4.21) is

$$
f=-g \sim \exp \left\{-\frac{q_{y} r^{\kappa \mathcal{R}}}{R_{y}}\right\}, r \rightarrow \infty
$$

This profile is essentially the same as (2.15) if we relate the coordinates $r$ and $\rho$

$$
\rho=r^{\kappa \mathcal{R}}
$$

In the core of the brane the functions $\mu(r), \lambda(r)$ behave as in (4.2) and the profile (4.21) is

$$
f=-g \sim \exp \left\{-k \frac{m}{4 r}\right\}, r \rightarrow 0
$$

If $q_{y}>0, f$ and $g$ vanish both when $r \rightarrow \infty$ and $r \rightarrow 0$ and the profile (4.21) corresponds to a fermion state localized on the brane.

Let us suppose that the brane thickness $r_{0}$ is quite small, so that the region $r>r_{0}$ gives the main contribution in the normalization (2.16) of the fermion modes. If the metric outside the brane is given by (4.5), the normalized solution of Dirac equation which describes an $S U(2)$ singlet state is given by (see (4.18), (4.21))

$$
F_{q, 0,0}=\frac{\sqrt{\left|q_{y}\right|}}{\sqrt{\pi} R_{y}} e^{-\left|q_{y}\right|\left(\rho-\rho_{0}\right) / R_{y}}\left(\begin{array}{c}
1 \\
i
\end{array}\right) \otimes \eta_{1 / 2,0,0}(\theta, \phi)
$$

while the $S U(2)$ doublet state has the profile

$$
F_{q, 1 / 2, m}=\frac{\sqrt{|q|}}{\sqrt{\pi} R_{y}} e^{-\left|q_{y}\right|\left(\rho-\rho_{0}\right) / R_{y}}\left(\begin{array}{c}
1 \\
i
\end{array}\right) \otimes \eta_{1,1 / 2, m}(\theta, \phi)
$$

If the monopole field (4.6) appears as a Kaluza-Klein field (4.9), then the charges $q_{y}$ and $e$ are related through (4.11) and instead of the whole tower of singlet and doublet states (4.25), (4.26) with different $q_{y}=1,2, \ldots$, we get just one singlet with $q_{y}=1$ and the only doublet with $q_{y}=2$. 
The effective four-dimensional Dirac masses of localized fermions can be calculated by the same procedure as in the previous section. The conserved angular momentum for the Higgs scalar in the field of magnetic monopole (4.6) is given by (4.7) without the spin operator $\frac{1}{2} \vec{\sigma}$. If the charge of the Higgs field is $e_{h}=1 /(2 m)$ the lowest possible value of the angular momentum is $j_{h}=1 / 2$. Therefore the higher-dimensional profile of the Higgs field is

$$
H_{p, j=1 / 2, m=1 / 2}=H_{p}(r) e^{i p y / R_{y}} Y_{1 / 2,1 / 2,1 / 2}(\theta, \phi)
$$

where $Y_{1 / 2,1 / 2,1 / 2}$ is the corresponding monopole harmonic [21]. In the same way as in (3.8) the Dirac mass of a fermion is given by the integral over the extra dimensions of the overlap of the singlet (4.25) and doublet (4.26) profiles with the profile of the Higgs field (4.27)

$$
M_{D}=f \int d r d y d \theta d \phi e^{(\lambda-\nu) / 2} H_{p, 1 / 2,1 / 2} \bar{F}_{q_{R}, 0,0} F_{q_{L}, 1 / 2, m}
$$

Integrating over the angles $(\theta, \phi)$ we find that the mixing of the doublet state with projection of angular momentum $m=-1 / 2$ with the singlet vanishes. The integral (4.28) for the mixing of $m=1 / 2$ component of the doublet with the singlet reduces to (3.10). The mass $M_{D}$ is expressed through the charges $q_{L}$ and $q_{R}$ of doublet (left) and singlet (right) components in the same way as in the previous Section. Depending on the profile of the Higgs field $H_{p}$ we can get either hierarchical (3.13) or quasidegerate (3.11) pattern of fermion masses.

It is straightforward to generalize the above model to the case when the brane is embedded in the nine-dimensional space-time with asymptotic $A d S_{7} \times S^{2}$ geometry. In this case the localized fermion modes are charged with respect to the $S U(2) \times U(1) \times U(1)$ symmetry group. One of the $U(1)$ factors can be related to the $U(1)_{Y}$ gauge group of the standard model, while the other will correspond to the horizontal symmetry $U(1)_{G}$. There are $S U(2)$ singlets and doublets in the spectrum of localized fermions. After the symmetry breaking the upper components of doublets become massive. The masses of these states are proportional to different powers of a small parameter $\epsilon$ (3.20), (3.21) and have hierarchical structure. Correspondingly, these states can be identified with the charged leptons of the standard model. The lower components of the $S U(2)$ doublets remain massless, since they are not mixed to the states localized on the brane. These states should be identified with neutrinos.

In our model the higher-dimensional profiles of the fermions are completely determined by their quantum numbers. In order to include quarks in the model we must equip the fermions with $S U(3)$ quantum numbers. We leave the discussion of relation between quark masses and quantum numbers for the future work.

\section{CONCLUSION.}

In this paper we have pointed out that in the models with a brane universe embedded into a higher-dimensional space-time, the fermions localized on the brane always possess quantum numbers which are their rotation momenta around the brane. We have considered the cases where the localized fermions carry $U(1)$ (Section [II), $U(1) \times U(1)$ (Section III) or $S U(2) \times U(1)($ Section $\mathbb{I V})$ quantum numbers. 
The higher-dimensional profiles of the fermions depend on their quantum numbers (2.15), and it turns out that the fermions which are neutral with respect to the group of rotations around the brane are not localized.

The effective four-dimensional masses of localized fermions are proportional to the overlaps of the profiles $\Psi_{L}, \Psi_{R}$ of left and right handed components of the fermion with the profile $H_{p}$ of (effective or fundamental) Higgs field (3.8). If the Higgs field is sharply peaked at a distance $\rho_{h}$ from the center of the brane, like in (3.12), the the masses of fermions with different quantum numbers go like different powers of a small parameter $\epsilon$, (3.20), (3.21). Even if there is no hierarchy in the length scales $\rho_{h}$ and $R_{z}$ in (3.21), the masses of localized fermions are arranged hierarchically.

\section{ACKNOWLEDGMENT.}

I am grateful to A.Barvinsky, X.Calmet, V.Mukhanov, I.Sachs and S.Solodukhin for useful discussions of the subject of the paper. This work was supported by the SFB 375 Grant of Deutsche Forschungsgemeinschaft. 


\section{REFERENCES}

[1] H. Fritzsch and Z. Xing, Prog. Part. Nucl. Phys. 45, 1 (2000) hep-ph/9912358.

[2] Y. Fukuda et al. [Super-Kamiokande Collaboration], Phys. Lett. B 436, 33 (1998) [hepex/9805006]; Y. Fukuda et al. [Super-Kamiokande Collaboration], Phys. Rev. Lett. 81, 1158 (1998) [Erratum-ibid. 81, 4279 (1998)] [hep-ex/9805021].

[3] C. D. Froggatt and H. B. Nielsen, Nucl. Phys. B 147, 277 (1979).

[4] R. N. Mohapatra, "Theories of neutrino masses and mixings," hep-ph/9910365.

[5] G. Dvali and A. Y. Smirnov, Nucl. Phys. B 563, 63 (1999) hep-ph/9904211; K. R. Dienes, E. Dudas and T. Gherghetta, Nucl. Phys. B 557, 25 (1999) hep-ph/9811428; N. Arkani-Hamed, S. Dimopoulos, G. Dvali and J. March-Russell, "Neutrino masses from large extra dimensions," hep-ph/9811448.

[6] N. Arkani-Hamed and M. Schmaltz, Phys. Rev. D 61, 033005 (2000) [hep-ph/9903417;

E. A. Mirabelli and M. Schmaltz, Phys. Rev. D 61, 113011 (2000) hep-ph/9912265;

M. Bando, T. Kobayashi, T. Noguchi and K. Yoshioka, Phys. Rev. D 63, 113017 (2001) hep-ph/0008120.

[7] G. Dvali and M. Shifman, Phys. Lett. B 475, 295 (2000) [hep-ph/0001072];

[8] A. Neronov, "Localization of Kaluza-Klein gauge fields on a brane," hep-th/0102210, to appear in Phy. Rev. D.

[9] P. G. Freund and M. A. Rubin, Phys. Lett. B 97, 233 (1980).

[10] M. J. Duff, B. E. Nilsson and C. N. Pope, Phys. Rept. 130, 1 (1986).

[11] J. Maldacena, Adv. Theor. Math. Phys. 2, 231 (1998) [Int. J. Theor. Phys. 38, 1113 (1998)] hep-th/9711200; E. Witten, Adv. Theor. Math. Phys. 2, 253 (1998) hepth/9802150].

[12] L. Randall and R. Sundrum, Phys. Rev. Lett. 83, 4690 (1999) hep-th/9906064.

[13] T. Gherghetta and M. Shaposhnikov, Phys. Rev. Lett. 85, 240 (2000) hep-th/0004014;

A. G. Cohen and D. B. Kaplan, Phys. Lett. B 470, 52 (1999) hep-th/9910132; R. Gregory, Phys. Rev. Lett. 84, 2564 (2000) hep-th/9911015.

[14] G. Dvali, S. Randjbar-Daemi and R. Tabbash, "The origin of spontaneous symmetry breaking in theories with large extra dimensions," hep-ph/0102307; M. Shaposhnikov and P. Tinyakov, "Extra dimensions as an alternative to Higgs mechanism?," hepth/0102161.

[15] M. V. Libanov and S. V. Troitsky, Nucl. Phys. B 599, 319 (2001) hep-ph/0011095.

[16] R. Jackiw and P. Rossi, Nucl. Phys. B 190, 681 (1981).

[17] A. Neronov, "Brane world cosmological constant in the models with large extra dimensions," gr-qc/0101060.

[18] S. Randjbar-Daemi, A. Salam and J. Strathdee, Nucl. Phys. B 214, 491 (1983).

[19] X. Calmet and A. Neronov, "Kaluza-Klein theories and the anomalous magnetic moment of the muon," hep-ph/0104278.

[20] Y. Kazama and C. N. Yang, Phys. Rev. D 15, 2300 (1977).

[21] T. T. Wu and C. N. Yang, Nucl. Phys. B 107, 365 (1976). 